\title{
Juvenile xanthogranuloma: Awakening of the other histiocytosis?
}

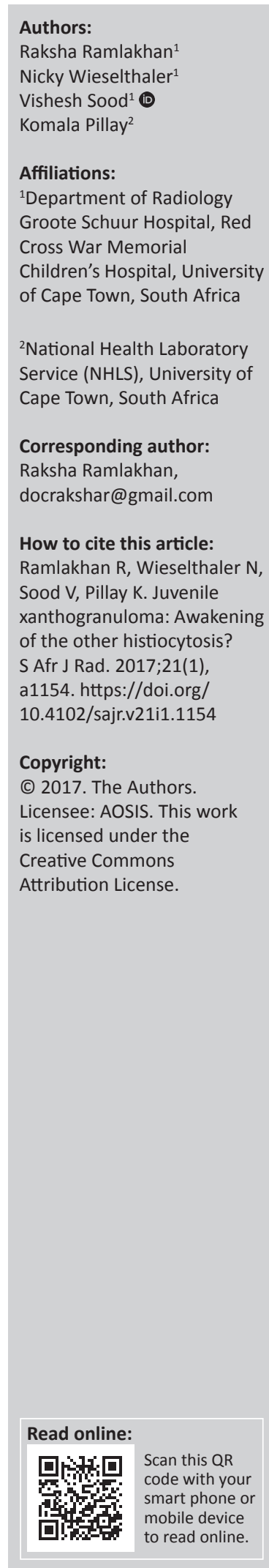

Juvenile xanthogranuloma is the lesser known and less innocuous of the histiocytic disorders. This non-Langerhans cell histiocytic disorder, as we know it, is a benign proliferative disorder manifesting as cutaneous lesions in young children. Extracutaneous lesions are less common with most systemic lesions reported in the liver, spleen, lung, eye, central nervous system and bones. These lesions usually spontaneously regress, or if not, treatment is based on severity and includes resection, chemotherapy and radiation. Mortality is rare. We present a case series of three patients presenting to Red Cross Children's Hospital over a 2-year period. We discuss the variable clinical presentation, the diverse imaging findings and the confirmatory histopathological diagnoses and treatment. We describe a cutaneous thigh lesion with visceral involvement. This poster highlights the first reported solitary bone lesion in the ulna. It also emphasises the aggressive and lethal nature of a cervical spine lesion. We demonstrate the difficulty in radiologically distinguishing these lesions from other tumours. Note: A selection of conference abstracts: RSSA/SASPI Paediatric Imaging Congress, 03-06 November 2016, Spier Estate, Stellenbosch,
South Africa. Faculty collaborators: Professor Kassa Darge (Body Imaging, University of Pennsylvania, Philadelphia, USA), Professor South Africa. Faculty collaborators: Professor Kassa Darge (Body Imaging, University of Pennsylvania, Philadelphia, USA), Professo USA), Professor Kimberly Applegate (Image Gently and Body Imaging, Emory University, Atlanta, USA) and Professor Savvas Andronikou (Thoracic Imaging, University of Bristol, UK) supported by South African Paediatric Radiologists, co-ordinated by Dr Jaishree Naidoo, President of the African Society of Paediatric Imaging and Head of Division of Paediatric Radiology, Charlotte Maxeke Johannesburg Academic Hospital. 